\title{
Untersuchungen über die Plasteinbildung.
}

\section{Mitteilung.}

Von

V. Henriques und J. K. Gjaldbæk.

(Der Redaktion zugegangen am 13. September 1912.)

In einem früheren Aufsatz ${ }^{1}$ ) berichteten wir über die Ergebnisse einiger Versuche über die Plasteinbildung durch Einwirkung von Pepsinsalzsäure auf konzentrierte Lösungen peptischer Spaltungsprodukte, wobei wir als Untersuchungsmethoden die Sörensensche Formoltitrierung und die Gerbsäurefällung benutzten; wir geben hier kurz die von uns gefundenen Verhältnisse an.

Wird eine konzentrierte Lösung eines peptischen Spaltungsprodukts mit Pepsinsalzsäure im Thermostaten angebracht, finden gleichzeitig mit der Plasteinbildung 1. eine Abnahme des formoltitrierbaren Stickstoffs und 2. eine Vermehrung des mit Gerbsäure fällbaren Stickstoffs statt. Die Größe der vorkommenden Veränderungen hängt von der Beschaffenheit des Ausgangsmaterials ab, indem der am stärksten abgebaute Stoff die größten Ausschläge in den erwähnten Richtungen ergibt.

Wir werden nun im folgenden über einige neue Versuche über die Plasteinbildung berichten. Wie man ersehen wird, hielten wir uns bei der Entscheidung der Frage «Synthese* ausschließlich an die Formoltitrierung und unternahmen keine Gerbsäurefällungen; dagegen wurde das Verhalten des Ammoniaks während der Plasteinbildung berücksichtigt.

Die Untersuchungsmethode war dieselbe wie bei unseren früheren Untersuchungen über die Plasteinbildung, indem die konzentrierte Lösung des Spaltungsprodukts + Ferment im Verhältnis $1: 20$ mit ausgekochtem, destilliertem Wasser gemischt wurde;

1) V. Henriques und J. K. Gjaldbæk, Diese Zeitschrift, Bd. 71. 
in der so zubereiteten Mischung wurde die Formoltitrierung ohne vorhergehende Neutralisation gegenüber Lackmuspapier (zur Vermeidung von Neutralisationsfehlern) unternommen und das Ammoniak durch Destillation im Vakuum mit Baryumhydroxyd, das in Methylalkohol gelöst war, bestimmt. Die gefundenen Werte wurden nach dem Stickstoffgehalt der Mischung reduziert, so daß sie $100 \mathrm{mg} \mathrm{N}$ entsprachen; der Stickstoffgehalt der Menge der Mischung (50 ccm), in welcher die Formoltitrierung und die Ammoniakbestimmungen stattfanden, schwankte um ca. $135 \mathrm{mg} \mathrm{N}$. Die Formoltitrierung ist angegeben in Kubikzentimetern ${ }^{1 / 5}-\mathrm{n}-\mathrm{NaOH}$, und in ihr ist - was die salzsauren Mischungen betrifft - die enthaltene freie Säure mit einbegriffen; das Ammoniak ist in Milligramm $N$ angegeben. Schließlich wurde der Stickstoffgehalt des beim Prozesse gebildeten Plasteins bestimmt, indem Stickstoffbestimmungen 1 . in $10 \mathrm{ccm}$ der aufgeschlämmten Versuchsmischung und 2 . in $10 \mathrm{ccm}$ von deren Filtrat unternommen wurden.

\section{Das Verhalten peptischer Spaltungsprodukte gegenüber Pepsin.}

Die Plasteinbildung durch Einwirkung der Pepsinsalzsäure auf peptische Spaltungsprodukte haben wir bereits früher untersucht $;^{1)}$ aus unseren früheren Untersuchungen ging hervor, daß Witte-Pepton, das 14\% formoltitrierbaren Stickstoffs enthält, Ausschlag in der Richtung einer Synthese ergab, die $0,65 \%$ formoltitrierbarem $\mathrm{N}$ entsprach, während peptische Spaltungsprodukte von Witte-Pepton, Casein und Hühnereiweiß (bis zu einem Gehalt von ca. 20\% formoltitrierbaren Stickstoffs verdaut) Ausschläge von 1,12-1,96\% formoltitrierbarem N ergaben. Ein pepsinverdautes Plasteinpräparat (bis zu einem Gehalt von $27 \%$ formoltitrierbaren Stickstoffs verdaut) ergab einen Ausschlag von 2,36\% formoltitrierbarem $\mathrm{N}$.

Da man indessen bei andauernder Pepsinverdauung ${ }^{2}$ ) einen bedeutend größeren Spaltungsgrad erreichen kann als $27 \%$, und da eben die am stärksten abgebauten Stoffe die

1) a. a. 0 .

2) V. Henriques und J. K. Gjaldbæk, Diese Zeitschrift, Bd. 75. 
größten Ausschläge ergaben, unternahmen wir einige Versuche mit stärker gespaltenen Stoffen. Ferner haben wir von anderen verdauten Proteinen Edestin, Gelatine und Rindfleisch herangezogen.

Wir verzeichnen unten den Spaltungsgrad, Ammoniakgehalt und das Verhältnis zwischen dem 1. und 4. Stadium bei der Formoltitrierung: $1-(4 \div \mathrm{k})$ für die angewandten Stoffe.

\begin{tabular}{|c|c|c|c|c|c|c|}
\hline & & & $\left|\begin{array}{c}\text { Spal- } \\
\text { tungs- } \\
\text { grad } \\
\text { (formol- } \\
\text { titrier- } \\
\text { barer N } \\
\text { in \% } \% \text { des } \\
\text { Total-N) }\end{array}\right|$ & $\begin{array}{c}\text { Am- } \\
\text { moniak- } \\
\mathrm{N} \text { in } \% \\
\text { des } \\
\text { Total-N }\end{array}$ & $1-(4 \div \mathrm{k})$ & $\begin{array}{c}\text { Ange- } \\
\text { wandt } \\
\text { in Ver- } \\
\text { such }\end{array}$ \\
\hline \multicolumn{3}{|c|}{ Pepsin- $\mathrm{H}_{2} \mathrm{SO}_{4}$ verdautes Hühnereiweiß } & 38,0 & 7,5 & $1-3,0$ & I \\
\hline > & $>$ & Casein & 38,5 & 8,8 & $1-3,3$ & II \\
\hline , & $\triangleright$ & > mageres Rindfleisch & 34,4 & 4,9 & $1-2,5$ & III \\
\hline$\gg$ & $\triangleright$ & - Witte-Pepton & 37,0 & 5,7 & $1-3,0$ & IV \\
\hline ১ & $\mathrm{HCl}$ & D Witte-Pepton & 34,6 & 5,1 & $1-2,8$ & V \\
\hline$>$ & $\triangleright$ & , Edestin & 32,2 & 8,8 & $1-2,8$ & VI \\
\hline$>$ & $\triangleright$ & , mageres Rindfleisch & 34,6 & 6,2 & $1-2,5$ & VII \\
\hline s & > & - Gelatine & 24,5 & 1,3 & $1-3,1$ & \\
\hline
\end{tabular}

Bei der Zubereitung der bei den Versuchen benutzten Mischungen verfuhren wir in folgender Weise: Wenn bei der Pepsinverdauung Schwefelsäure angewandt wurde, würde die Säure mit Baryumhydroxyd aus den schwefelsauren Flüssigkeiten entfernt; wurde Salzsäure angewandt, wurde mit 1-n$\mathrm{NaOH}$ neutralisiert, wonach die Flüssigkeit filtriert wurde. Das neutral reagierende Filtrat wurde bis auf $100 \mathrm{ccm}$ (mit N-Gehalt von $7 \mathrm{~g}$ ) eingedampft; es wurden $20 \mathrm{ccm} \mathrm{1-n-HCl,} 20 \mathrm{ccm}$ $5 \%$ iger Pepsinlösung und Toluol hinzugesetzt. Sofort nach der Zusammenmischung wurden Untersuchungsproben entnommen, und der Rest wurde bei $37^{\circ}$ in den Thermostaten gestellt.

Bei den Versuchen I, II und III wurden peptische Spaltungsprodukte von Hühnereiweiß, Casein und Rindfleisch mit einem Gehalt von 38, 38,5 und $34,4 \%$ formoltitrierbarem $\mathrm{N}$ angewandt. 
Tabelle I.

Ca. 30\%ige Lösung von Pepsin- $\mathrm{H}_{2} \mathrm{SO}_{4}$-verdautem Hühnereiweiß + Pepsin-HCl.

\begin{tabular}{|c|c|c|c|c|}
\hline & $\begin{array}{c}\text { Formol- } \\
\text { titrierung } \\
\text { pro } 100 \mathrm{mg} \mathrm{N} \\
\mathrm{ccm} 1 / \mathrm{s}-\mathrm{n}- \\
\mathrm{NaOH}\end{array}$ & $\begin{array}{c}\text { Am- } \\
\text { moniak-N } \\
\text { in } \% \text { des } \\
\text { Total-N }\end{array}$ & $\left|\begin{array}{c}\text { Plastein-N } \\
\text { in } \% \\
\text { des } \\
\text { Total-N }\end{array}\right|$ & $\begin{array}{l}\text { Physikalische } \\
\text { Änderungen }\end{array}$ \\
\hline Sogleich . . . . . & 15,1 & 7,6 & 0 & Klare Lösung. \\
\hline 1 Stunde bei $37^{\circ}$. & 14,9 & - & 2,6 & $\begin{array}{l}\text { Deutliche Aus- } \\
\text { fällung. }\end{array}$ \\
\hline 20 Stunden bei $37^{\circ}$. & 14,2 & - & 17,9 & \\
\hline 2 tägiges Stehen bei $37^{\circ}$ & 14,4 & - & 18,1 & Große \\
\hline $4=, 37^{\circ}$ & 14,2 & - & 18,7 & Ausfällung, \\
\hline $11, \quad \times 37^{\circ}$ & 13,7 & - & 23,6 & aber keine \\
\hline Zusatz von $1 \mathrm{~g}$ Pepsin & & & & Gela- \\
\hline Sogleich nach Zusatz . & 13,7 & - & - & tinierung. \\
\hline 14 tägiges Stehen bei $37^{\circ}$ & 13,7 & 7,5 & 24,9 & \\
\hline
\end{tabular}

Tabelle II.

Ca. $30 \%$ ige Lösung von Pepsin- $\mathrm{H}_{2} \mathrm{SO}_{4}$-verdautem Casein + Pepsin-HGl.

\begin{tabular}{|c|c|c|c|c|}
\hline & $\begin{array}{l}\text { Formol- } \\
\text { titrierung } \\
\text { pro } 100 \mathrm{mg} \mathrm{N} \\
\text { ccm } 1 / 5-\mathrm{n}- \\
\mathrm{NaOH}\end{array}$ & $\begin{array}{l}\text { Am- } \\
\text { moniak-N } \\
\text { in } \% \text { des } \\
\text { Total-N }\end{array}$ & $\begin{array}{c}\text { Plastein-N } \\
\text { in } \% \\
\text { des } \\
\text { Total-N }\end{array}$ & $\begin{array}{l}\text { Physikalische } \\
\text { Änderungen }\end{array}$ \\
\hline Sogleich . . . . . & 15,3 & 8,2 & 0 & Klare Lösung. \\
\hline 1 Stunde bei $37^{\circ}$. & 15,1 & - & 0,8 & $\begin{array}{l}\text { Sichtbare Aus- } \\
\text { fällung. }\end{array}$ \\
\hline 20 Stunden bei $37^{\circ}$ & 14,8 & - & 9,8 & \\
\hline 2 tägiges Stehen bei $37^{\circ}$ & 14,6 & - & 13,3 & Große \\
\hline $4,>>37^{\circ}$ & 14,5 & 一 & - & Ausfällung, \\
\hline$>\quad 37^{\circ}$ & 14,3 & - & 15,4 & aber keine \\
\hline Zusatz von 1 g Pepsin & & & & Gela- \\
\hline Sogleich nach Zusatz . & 14,3 & - & - & tinierung. \\
\hline 14 tägiges Stehen bei $37^{\circ}$ & 14,4 & 8,1 & - & \\
\hline
\end{tabular}


Tabelle III.

Pepsin- $\mathrm{H}_{2} \mathrm{SO}_{4}$-verdautes Rindfleisch in $30 \%$ iger Lösung + Pepsin-HCl.

\begin{tabular}{|c|c|c|c|c|}
\hline & $\begin{array}{c}\text { Formol- } \\
\text { titrierung } \\
\text { pro } 100 \mathrm{mg} \mathrm{N} \\
\text { ccm } 1 / 5-\mathrm{n}- \\
\mathrm{NaOH}\end{array}$ & $\begin{array}{c}\text { Am- } \\
\text { moniak-N } \\
\text { in } \% \text { des } \\
\text { Total-N }\end{array}$ & $\left|\begin{array}{c}\text { Plastein-N } \\
\text { in } \% \\
\text { des } \\
\text { Total-N }\end{array}\right|$ & $\begin{array}{c}\text { Physikalische } \\
\text { Änderung }\end{array}$ \\
\hline Sogleich . . . . . & 14,1 & 4,7 & - & Klare Lösung. \\
\hline 1 Stunde bei $37^{\circ} \ldots$ & 13,8 & 一 & 2,0 & $\begin{array}{l}\text { Deutliche Aus- } \\
\text { fällung. }\end{array}$ \\
\hline 20 Stunden bei $37^{\circ}$. & 13,6 & - & 10,5 & \\
\hline 2 tägiges Stehen bei $37^{\circ}$ & 13,8 & - & 10,5 & Große \\
\hline $4 \gg>37^{\circ}$ & 13,5 & - & 12,6 & Ausfällung, \\
\hline $11>>37^{\circ}$ & 13,4 & 一 & 15,1 & aber keine \\
\hline Zusatz von 1 g Pepsin & & & & Gela- \\
\hline Sogleich nach Zusatz . & 13,4 & 一 & - & tinierung. \\
\hline 14 tägiges Stehen bei $37^{\circ}$ & 13,5 & 4,9 & 15,3 & \\
\hline
\end{tabular}

Wie man sieht, ist die Plasteinbildung in allen Fällen sichtbar nach 1 Stunde, und im Laufe dieser Zeit wurden bezw. 2,6, 0,8 und 2,0\% des Stickstoffgehalts der Mischungen als Plastein ausgefällt; gleichzeitig beobachtet man auch eine Abnahme des formoltitrierbaren $N$. Bei längerem Stehen im Thermostaten schreitet der Prozeß weiter fort, und wenn er erst ängehalten hat, scheint ein neuer Zusatz von Pepsin ohne Wirkung zu sein.

Bei den Versuchen I und II hörte die Abnahme des formoltitrierbaren $\mathrm{N}$ erst nach 11 Tagen auf; bei dem Versuch III dagegen erreichte die Abnahme des formoltitrierbaren $\mathrm{N}$ ihr Maximum bereits nach 1 Tage; die Ausfällung von Plastein hört in diesem Falle nicht gleichzeittg mit der Erreichung des genannten Maximums auf, was darauf deutet, daß die Synthese und die Fällung zwei verschiedene und ungleich schnell verlaufende Stadien des Prozesses oder vielleicht zwei parallele, aber voneinander unabhängige Prozesse sind. Bei Beendigung der Versuche waren bezw. 24,9, 15,4 und 15,3\% 
des Total-N der Mischung als Plastein ausgefällt. Bei keinem Versuch trat Gelatinierung ein, aber die Fällung war, wie aus den angeführten Zahlen ersichtlich, recht bedeutend.

Die Abnahme des formoltitrierbaren $\mathrm{N}$ beträgt bei $\mathrm{Be}-$ endigung der Versuche bezw. 1,4, 1,0 und $0,6 \mathrm{~cm}{ }^{1} / 5-\mathrm{n}-\mathrm{NaOH}$ pro $100 \mathrm{mg} \mathrm{N}$. Die Zahlen entsprechen also 3,92, 2,8 und $1,68 \%$ formoltitrierbarem $\mathrm{N}$.

Die Ammoniakmenge blieb bei allen drei Versuchen unverändert.

Das. gebildete Plastein war bei allen drei Versuchen fast, wenn auch nicht vollständig löslich in schwachem $\mathrm{NaOH}$ und schwachem $\mathrm{HCl}$.

Tabelle IV.

Ca. $30 \%$ ige Lösung von Pepsin- $\mathrm{H}_{2} \mathrm{SO}_{4}$-verdautem

Witte-Pepton + Pepsin-HCl.

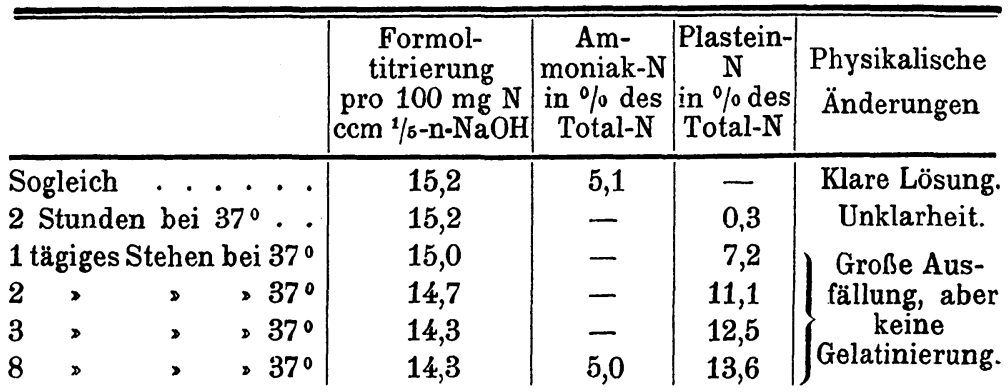

Tabelle V.

Ca. $30 \%$ ige Lösung von Pepsin-HGl-verdautem Witte-Pepton + Pepsin-HCl.

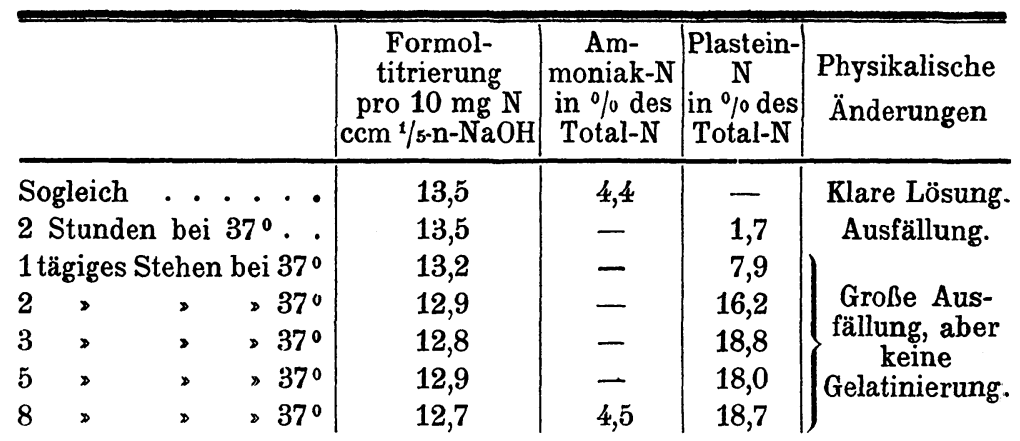


Tabelle VI.

Ca. 30\%ige Lösung von Pepsin-HCl-verdautem Edestin + Pepsin-HCl.

\begin{tabular}{|c|c|c|c|c|c|}
\hline \multicolumn{2}{|c|}{. } & $\begin{array}{c}\text { Formol- } \\
\text { titrierung } \\
\text { pro } 100 \mathrm{mg} \mathrm{N} \\
\text { ccm } 1 / \mathrm{s}-\mathrm{n} \cdot \mathrm{NaOH}\end{array}$ & $\begin{array}{c}\text { Am- } \\
\text { moniak-N } \\
\text { in } \% \text { des } \\
\text { Total-N }\end{array}$ & $\mid \begin{array}{c}\text { Plastein- } \\
\mathrm{N} \\
\text { in } \% \text { des } \\
\text { Total-N }\end{array}$ & $\begin{array}{l}\text { Physikalische } \\
\text { Änderungen }\end{array}$ \\
\hline \multirow{2}{*}{\multicolumn{2}{|c|}{$\begin{array}{l}\text { Sogleich } \\
2 \text { Stunden bei } 37^{\circ}\end{array}$}} & 13,3 & 7,9 & - & \multirow{7}{*}{$\begin{array}{l}\text { Klare Lösung } \\
\text { Ausfällung. } \\
\text { Große Aus- } \\
\text { fällung, aber } \\
\text { keine } \\
\text { Gelatinierung }\end{array}$} \\
\hline & & 13,1 & - & 2,7 & \\
\hline & giges Stehen bei $37^{\circ}$ & 12,6 & - & 11,5 & \\
\hline 2 & $=\quad, 37^{\circ}$ & 12,4 & - & 12,1 & \\
\hline 3 & $\triangleright \quad .37^{\circ}$ & 12,3 & - & 18,1 & \\
\hline 5 & $>\quad>37^{\circ}$ & 12,4 & - & 18,1 & \\
\hline 8 & $>\quad=37^{\circ}$ & 12,3 & 7,9 & 18,3 & \\
\hline
\end{tabular}

Tabelle VII.

Ca. 30\%ige Lösung von Pepsin-HCl-verdautem Rindfleisch + Pepsin-HGl.

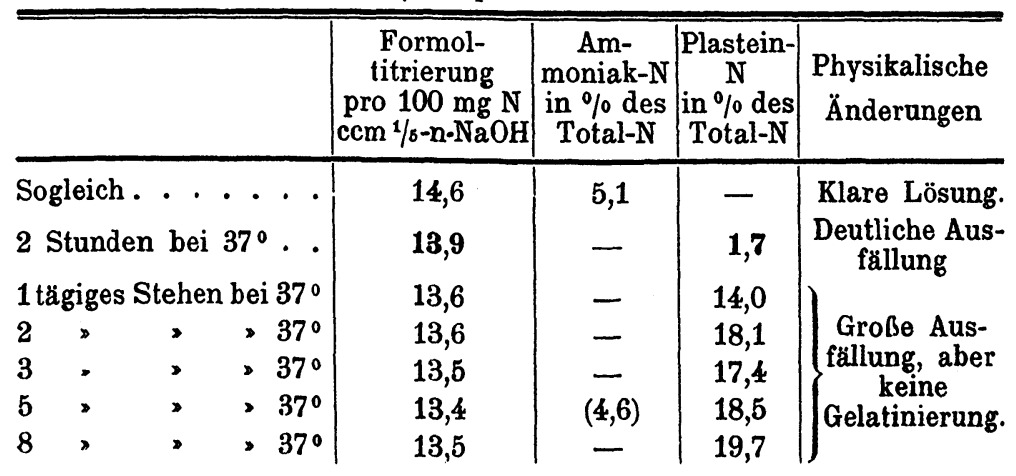

Bei den Versuchen IV-VII wurden Pepsin- $\mathrm{H}_{8} \mathrm{SO}_{4}$-verdautes Wit te-Pepton, Pepsin-HCl-verdautes Wit te-Pepton, Edestin und Rindfleisch mit einem Spaltungsgrad von bezw. $37,0,34,6,32,2$ und $34,6 \%$ formoltitrierbarem $\mathrm{N}$ angewandt.

Die Versuchsresultate entsprechen in der Hauptsache denen der Versuche I-III; die Plasteinausfällung und die Abnahme des formoltitrierbaren $\mathrm{N}$ begannen bereits nach 2 Stunden, und die Prozesse setzten sich in jedem Falle einige Tage hindurch fort. Bei den Versuchen V, VI und VII hörte die Abnahme des formoltitrierbaren $\mathrm{N}$ vor der Plasteinaus- 
fällung auf (vgl. Versuch III), was, wie erwähnt, darauf deutet, daß die Synthese und die Ausfällung zwei verschiedene und ungleich schnell verlaufende Stadien des Prozesses oder vielleicht zwei parallele, aber voneinander unabhängige Prozesse sind, indem die Synthese vor der Ausfällung stattfindet. Daß die Ausfällung und die Synthese nicht gleichzeitig stattfinden, sieht man deutlich aus dem Versuche VII. Hier fand im Laufe von 2 Stunden eine 1,96\% formoltitrierbarem $\mathrm{N}$ entsprechende Synthese statt, während die Plasteinausfüllung nur $1,7 \%$ des Total-N betrug; nach 1 Tage entsprach aber die Synthese $2,8 \%$, während die Plasteinausfällung $14 \%$ betrug. Diese Zahlen zeigen, daß eine nicht unbedeutende Synthese ohne wesentliche Plasteinausfällung stattfinden kann. Bei keinem Versuch trat Gelatinierung ein, die Plasteinausfällung war aber recht bedeutend: $13,6-19,70 \%$ des Stickstoffgehalts der Mischungen. Das gebildete Plastein war bei allen Versuchen fast, aber nicht vollständig löslich in schwachem $\mathrm{NaOH}$ und schwachem $\mathrm{HCl}$.

Die Abnahme des formoltitrierbaren $\mathrm{N}$ beträgt bezw. $0,9,0,8,1,0$ und $1,2 \mathrm{ccm} 1 / 5-\mathrm{n}-\mathrm{NaOH}$, was (durch Multiplikation mit 2,8) in formoltitrierbaren $\mathrm{N}$ umgesetzt, 2,52, 2,24, 2,8 und $3,36 \%$ entspricht.

Die Ammoniakmenge blieb unverändert.

Bei Beendigung der Versuche IV-VII fand wie gewöhnlich eine Formoltitrierung in $50 \mathrm{ccm}$ einer wässerigen Ausreibung der Versuchmischungen statt; um aber zu versuchen, ob der Prozeß bei Verdünnung wieder zurückgeht, wurden $\mathrm{zu}$ anderen $50 \mathrm{ccm} 5 \mathrm{ccm} 1-\mathrm{n}-\mathrm{HCl}$ gesetzt; diese ca. 1/10-n-Salzsäure (und pepsinhaltige) Flüssigkeiten wurden 1 Tag in den Thermostaten gestellt, und es wurden ihnen sodann $5 \mathrm{ccm} \mathrm{n}-\mathrm{NaOH}$ hinzugesetzt, worauf eine Formoltitrierung stattfand. Das Ergebnis dieser Versuche war: Das Plastein löste sich nach und nach und war nach 1 Tage vollständig gelöst; gleichzeitig stieg der Formoltiter und erreichte eine Größe, die sehr annäherungsweise dem von der Versuchsmischung vor der Plasteinbildung aufgewiesenen Formoltiter entsprach. Wir führen hier die Zahlen an. 


\begin{tabular}{l|l|c|c|c|c}
\hline \hline & $\begin{array}{c}\text { Ver- } \\
\text { such } \\
\text { IV }\end{array}$ & $\begin{array}{c}\text { Ver- } \\
\text { such }\end{array}$ & $\begin{array}{c}\text { Ver- } \\
\text { such } \\
\text { VI }\end{array}$ & $\begin{array}{c}\text { Ver- } \\
\text { such } \\
\text { VII }\end{array}$ \\
\hline Formoltiter vor der Plasteinbildung pro $100 \mathrm{mg} \mathrm{N}$ & 15,2 & 13,5 & 13,3 & 14,6 \\
nach , $, 100,>$ & 14,3 & 12,7 & 12,3 & 13,4 \\
Formoltiter nach der Plasteinbildung pro 100 mg N & & & & \\
und darauffolgender Verdünnung mit 1/10-n-HCl & 14,9 & 13,6 & 13,2 & 14,2 \\
und 1 tägigem Stehen bei 370 & & &
\end{tabular}

Aus obenstehendem geht hervor, daB man sowohl bewirken kann, daß der Prozeß in der einen Richtung verläuft wie in der anderen, je nach der Konzentration, und die Versuche stellen also die Wirkung der Pepsinsalzsäure auf Proteine und peptische Spaltungsprodukte als einen reversiblen ProzeB dar.

Von sonstigen pepsinverdauten Proteinen haben wir mit pepsinverdauter Gelatine Versuche angestellt; hier kam aber keine Plasteinbildung vor; die Flüssigkeit hält sich vollkommen klar, und der Formoltiter hielt sich konstant.

Vergleicht man die Ergebnisse der obenerwähnten Versuche mit den Ergebnissen unserer früheren Versuche mit weniger stark gespaltenen Proteinen, so sieht man, daß die Plasteinbildung am kräftigsten vonstatten geht bei Anwendung von nicht zu stark gespaltenen Proteinen; so findet bei Anwendung von Witte-Pepton eine vollständige Gelatinierung oder Koagulation statt, so daß die zu Anfang klare und flüssige Mischung erstarrt, indem gleichzeitig ein Stoff ausgefällt wird. Werden dagegen stärker gespaltene Proteine angewandt, so bleibt die Mischung flüssig, aber es wird ein Stoff ausgefällt. Die Menge des ausgefällten Stoffs schwankte nicht stark bei den Versuchen mit stark gespaltenen und weniger stark gespaltenen Proteinen; doch scheint die Ausfällung durchgehends am größten bei Anwendung von stark gespaltenen Proteinen.

Und nun das Plastein selbst. Das aus sehr stark gespaltenen Proteinen gebildete Plastein weicht dadurch von dem 
aus weniger stark gebildeten $a b$, daß es in schwachem $\mathrm{NaOH}$ und schwachem $\mathrm{HCl}$ unvollständig löslich ist, was darauf deutet, daß das Plastein kein einfacher Stoff ist, sondern wahrscheinlich eine Mischung von Albumosen.

In betreff der Synthese bestätigen die Versuche deutlich die Ergebnisse unserer früheren Untersuchungen; die am stärksten abgebauten Stoffe geben die größte Synthese ab. Die größte Synthese fand sich bei Versuch I, wo die Veränderung an formoltitrierbarem Stickstoff ca. 4\% der Totalstickstoffmenge betrug, was einer Veränderung von gut $10 \%$ des formoltitrierbaren Stickstoffs entspricht, indem der Stoff $38 \%$ davon enthielt.

Erinnert man sich nun, daß ca. 25\% des Total-N hier als Plastein ausgefällt sind, und betrachtet man ferner das Plastein als das synthetisierte Produkt, so beträgt die Veränderung an formoltitrierbarem $\mathrm{N}$ ca. 40\% der gesamten formoltrierbaren Stickstoffmenge der zur Plasteinbildung verbrauchten Stoffe. Wie später besprochen werden wird, ist das Plastein indessen aus den am kompliziertesten gebauten Verbindungen des Ausgangsmaterials gebildet, und somit muß die Veränderung an formoltitrierbarem $\mathrm{N}$ für die Verbindungen, aus denen das Plastein gebildet ist, einen größeren Wert als $40 \%$ betragen.

Damit eine Synthese stattfinden kann, müssen sich mindestens zwei Polypeptide bei Anhydridbildung zwischen einer Carboxyl- und einer Aminogruppe zusammenschließen, was eine Veränderung von $50 \%$ des formoltitrierbaren $\mathrm{N}$ (in $\%$ des formoltitrierbaren $N$ ) bewirken wird, insofern die Polypeptide aus lauter Monaminosäuren bestehen; enthalten sie zugleich Diaminosäuren (was ja der Fall ist), wird die Veränderung weniger als $50 \%$ betragen.

Die Berechnung unserer Versuchsresultate ergibt also, $\mathrm{da} ß$ die Veränderung an formoltitrierbarem $\mathrm{N}$ für die an der Plasteinbildung beteiligten Stoffe mehr als $40 \%$ betragen muß, während eine Synthese eine Veränderung erfordert, die naturgemäß mehr als $50 \%$ betragen kann, die aber, wenn sich nur 2 Moleküle an der Synthese beteiligen, jedenfalls weniger als 
$50 \%$ betragen muß. Diese Berechnung stimmt sehr gut überein mit den gefundenen Verhältnissen und befürwortet in hohem Grade das Stattinden einer Synthese bei der Plasteinbildung.

Wie in unserer früheren Mitteilung über die Plasteinbildung berührt, gibt es bei der Plasteinbildung ein Verhältnis, von dem man annehmen könnte, daß es eine scheinbare Abnahme des formoltitrierbaren $\mathrm{N}$ bewirkte, nämlich die Ausfällung des Plasteins, indem man vor der Plasteinbildung in einer vollkommen klaren Lösung, während und nach derselben aber in einer Flüssigkeit titriert, die das Plastein in aufgeschlämmtem Zustand enthält, woraus man vermuten könnte, daß es während der Formoltitrierung nicht, oder jedenfalls nur unvollständig reagierte. Indessen tritt der größte Teil des Plasteins während der Formoltitrierung in Lösung, ein geringer Teil aber löst sich nicht. Um diesen eventuellen Fehler zu berücksichtigen, wurde nach Abschluß der Plasteinbildung in den verschiedenen Versuchen eine Aufschlämmung $(1: 20)$ in ausgekochtem, destilliertem Wasser zubereitet; die Aufschlämmung wurde gegenüber Lackmuspapier neutralisiert und der Formoltiter pro $100 \mathrm{mg} \mathrm{N}$ bestimmt. Ein anderer Teil der Aufschlämmung wurde filtriert, und der $100 \mathrm{mg}$ Filtrat-N und $100 \mathrm{mg}$ Plastein-N (das Plastein wurde, so weit möglich, vor der Formoltitrierung in der notwendigen Menge 1/5-n- $\mathrm{NaOH}+$ destill. Wasser gelöst) entsprechende Formoltiter wurde gleichfalls bestimmt, worauf der tatsächliche Formoltiter der Aufschlämmung aus dem Gehalt des Filtrats und Plasteins an formoltitrierbarem $\mathrm{N}$ berechnet wurde, indem der Plastein-N in $\%$ des Total- $\mathrm{N}$ bekannt war.

Der Vollständigkeit halber führten wir auch in gegenwärtigen Versuchen diese Bestimmungen aus und geben unten die Ergebnisse wieder, Aus ihnen ist ersichtlich, daß der auf direktem Wege gefundene Formoltiter mit dem durch Berechnung aus dem Formoltiter im Plastein und Filtrat gefundenen gut übereinstimmt, weshalb wir hier - wie bei unseren früheren Versuchen - folgern müssen, daß es mit keinem etwas bedeutenden Fehler verbunden ist, daß der auf direktem Wege bestimmte Formoltiter in einer Flüssigkeit bestimmt 
worden ist, die das Plastein in aufgeschlämmten Zustand enthält.

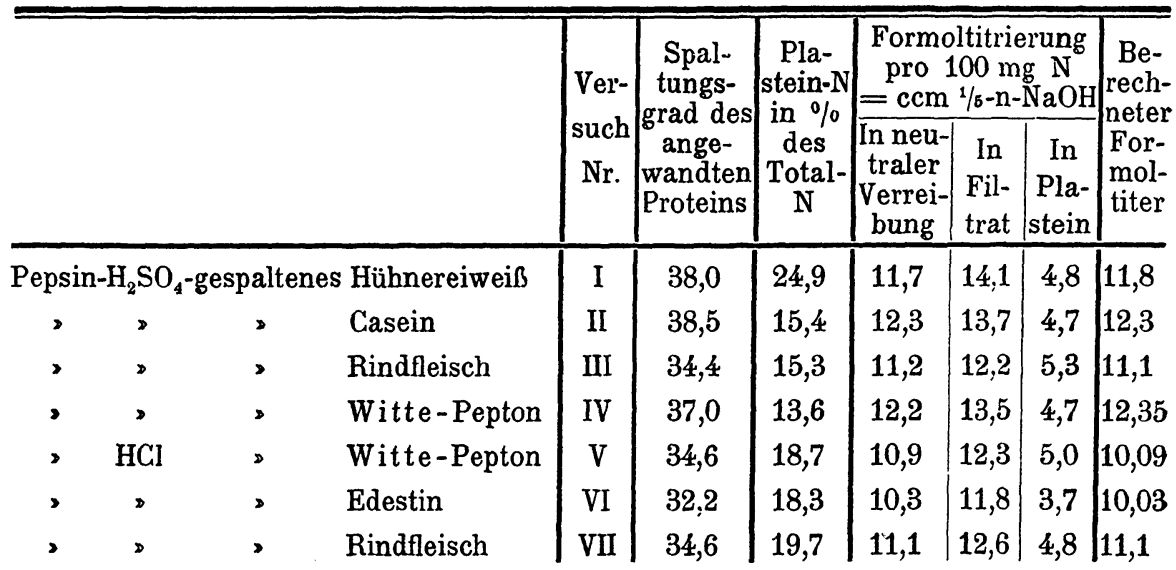

Aus obenstehender Tabelle läßt sich der Gehalt des Plasteins an formoltitrierbarem N (die Zahlen der zweitletzten Kolumne mit 2,8 multipliziert) leicht berechnen; er schwankt in den verschiedenen Versuchen zwischen 10,4 und 14,8\%, was etwas größere Zahlen sind als diejenigen, die man bei Anwendung von Witte-Pepton erhält; dagegen ergibt aus pepsinverdauten Proteinen hergestelltes Plastein mit einem Spaltungsgrad von ca. 20\% sozusagen dieselben Zahlen, wie Plastein aus den stärker gespaltenen Proteinen, die bei den oben angeführten Versuchen angewendet wurden. In betreff der in obenstehender Tabelle für den Spaltungsgrad des Plasteins angeführten Zahlen muß man sich jedoch erinnern, daß das Plastein in schwachem $\mathrm{NaOH}$ nicht ganz gelöst werden konnte, weshalb die gefundenen Werte möglicherweise etwas zu klein sind.

Aus der Tabelle geht ferner hervor, daß das Filtrat aus dem Plastein mehr formoltitrierbaren $\mathrm{N}$ enthält als das Ausgangsmaterial, woraus man schließen kann, daß das Plastein aus den am kompliziertesten gebauten Stoffen der Versuchsmischung gebildet ist.

In der 2. Kolumne der Tabelle ist der Spaltungsgrad des Ausgangsmaterials vor der Plasteinbildung angegeben. 
Ein Vergleich dieser Zahlen mit denen der Kolumne 4 (nach Multiplikation mit 2,8) gibt zugleich Aufschluß über die bei der Plasteinbildung stattgefundene Synthese; man muß sich aber erinnern, daß sowohl den Zahlen der 2. als denen der 4. Kolumne ein größerer oder kleinerer Neutralisationsfehler anhaftet, und der Umfang der bei der Plasteinbildung stattgefundenen Synthese darf somit nicht aus obenstehender Tabelle, sondern muß ausschließlich aus den Tabellen I-VI berechnet werden, wo den Zahlen keine Neutralisationsfehler anhaften.

Das Verhalten peptischer Spaltungsprodukte gegenüber Trypsin.

Wir haben früher mitgeteilt, daß Witte-Pepton in $30 \%$ iger neutraler Lösung typische Plasteinbildung mit Trypsin ergibt, während gleichzeitig eine starke Proteolyse stattfindet. Da Witte-Pepton aber nur $14 \%$ formoltitrierbaren $\mathrm{N}$ enthält, während die peptischen, in den oben angeführten Versuchen mit Pepsin angewandten Spaltungsprodukte fast $40 \%$ formoltitrierbaren $\mathrm{N}$ enthielten, meinten wir, daß Versuche mit diesen stark gespaltenen Stoffen vielleicht über den Prozeß der Plasteinbildung durch Trypsineinwirkung Aufschluß geben könnten.

Von den oben erwähnten peptischen Spaltungsprodukten, deren Analyse S. 441 angeführt ist, verwandten wir folgende: I Hühnereiweiß, II Casein, III Rindfleisch, VIII Gelatine und ferner eine Mischung von IV und V Witte-Pepton.

Die Versuchsmischungen wurden durch Eindampfung der neutralen Lösungen (von einem Gehalt von $7 \mathrm{~g} \mathrm{~N}$ ) zu $120 \mathrm{~g}$ hergestellt, zu denen $20 \mathrm{ccm} \mathrm{5 \%}$ \%iger Pankreatinlösung gesetzt wurden.

Beim Versuch mit Gelatine entstand keine Plasteinbildung, bei den übrigen Versuchen typische Plasteinbildung ohne Gelatinierung. Die Größe der Plasteinbildung betrug bei Beendigung der Versuche nach 15tägigem Stehen bei $37^{\circ}$ 15,9, 6,2, 5,6 und 11,4\% des Total-N bezw. für Hühnereiweiß, Casein, Rindfleisch und Witte-Pepton. Aus diesen Zahlen sieht man, 
daß die Plasteinausfällung bei den Versuchen mit Trypsin nicht so groß war, wie bei den Pepsinversuchen. Pepsinverdautes Hühnereiweiß ergibt hier, wie bei den Pepsinversuchen, die größte Plasteinausfällung.

Bei allen Versuchen fand eine Proteolyse statt; sie betrug bei Beendigung der Versuche (nach 15 Tagen bei $37^{\circ}$ ) 1,9, $1,7,2,7,2,0$ und 1,1 bezw. für Gelatine, Hühnereiweiß, Casein und Witte-Pepton; die Zahlen geben $1 / 3-\mathrm{n}-\mathrm{NaOH}$ pro $100 \mathrm{mg}$ $\mathrm{N}$ an. Indessen wiesen die Zahlen des Versuchs mit pepsinverdautem Hühnereiweiß Verhältnisse auf, die darauf deuten, daß die Plasteinbildung, außer von einer Proteolyse, von einem synthetischen Prozeß begleitet wird. Tabelle VIII berichtet über diesen Versuch.

Tabelle VIII.

Ca. $30 \%$ ige Lösung von Pepsin- $\mathrm{H}_{2} \mathrm{SO}_{4}$-verdautem Hühnereiweiß + Trypsin.

\begin{tabular}{|c|c|c|c|c|c|}
\hline & & $\begin{array}{c}\text { Formol- } \\
\text { titrierung } \\
\text { pro } 100 \mathrm{mg} \mathrm{N} \\
\text { ccm } 1 / 5-\mathrm{n}- \\
\mathrm{NaOH}\end{array}$ & $\begin{array}{c}\text { Am- } \\
\text { moniak-N } \\
\text { in } \% \text { des } \\
\text { Total-N }\end{array}$ & $\mid \begin{array}{c}\text { Plastein- } \\
\mathrm{N} \\
\text { in } \% \text { des } \\
\text { Total-N }\end{array}$ & Bemerkungen \\
\hline \multirow{2}{*}{\multicolumn{2}{|c|}{$\begin{array}{l}\text { Sogleich . . . } \cdot \cdot \cdot \cdot \cdot \\
1 \text { tägiges Stehen bei } 37^{\circ}\end{array}$}} & 13,5 & 5,5 & - & \multirow{5}{*}{$\begin{array}{l}\text { Klare Lösung. } \\
\text { Große Ausfällung, } \\
\text { aber keine } \\
\text { Gelatinierung; } \\
\text { sichtbare Tyrosin. } \\
\text { ausscheidung. }\end{array}$} \\
\hline & & 13,5 & - & 6,3 & \\
\hline 3 & $\triangleright \quad>37^{\circ}$ & 13,7 & - & 14,6 & \\
\hline 6 &,$\quad>37^{\circ}$ & 14,2 & 5,7 & 15,0 & \\
\hline 15 &,$\quad .37^{\circ}$ & 15,2 & 5,8 & 15,9 & \\
\hline
\end{tabular}

Aus dieser Tabelle sieht man, daß sich im Laufe des ersten Tages 6,3\% Plastein bilden, während der Formoltiter sich konstant hält; im Laufe der folgenden 2 Tage bilden sich ferner 8,3\% Plastein, während der Formoltiter um $0,2 \mathrm{ccm}$ $1 / 5-\mathrm{n}-\mathrm{NaOH}$ steigt; im Laufe der folgenden 3 Tage bilden sich $0,4 \%$ Plastein, und der Formoltiter steigt um 0,5 c $\mathrm{cm}^{1 / 5}-\mathrm{n}-\mathrm{NaOH}$, und im Laufe der letzten 9 Tage bilden sich $0,9 \%$ Plastein, während der Formoltiter um 1,0 ccm 1/5-n-NaOH steigt. Wir geben unten eine Übersicht über die Veränderung pro die in den verschiedenen Perioden der Analysen. 


\begin{tabular}{l|c|c}
\hline & $\begin{array}{c}\text { Vermehrung des formol- } \\
\text { titrierbaren N pro die }\end{array}$ & $\begin{array}{c}\text { Ausscheidung des Plasteins } \\
\text { pro die } \\
\text { (durchschnittliche Zahlen) }\end{array}$ \\
\hline 1. Tag . . . & 0 & $6,3 \%$ des Total-N \\
2. und 3. Tag. . & 0,1 & $4,15 \%$, \\
4., 5. und 6. Tag & 0,17 & $0,13 \%$, \\
7.-15. Tag inkl. & 0,11 & $0,10 \%$,
\end{tabular}

Die Vermehrung des formoltitrierbaren $\mathrm{N}$ geschieht in sehr eigentümlicher Weise; während die Plasteinbildung auf der Maximalstufe steht, die sie im Laufe des ersten Tages erreicht, findet keine Vermehrung des formoltitrierbaren $\mathrm{N}$ statt; je nachdem die Plasteinbildung abnimmt, setzt die Vermehrung des formoltitrierbaren $\mathrm{N}$ ein, und erst wenn die Plasteinbildung sozusagen aufgehört hat, erreicht die Vermehrung des formoltitrierbaren $\mathrm{N}$ ihr Maximum, um sodann schließlich abzunehmen. Man muß aus diesem Verhältnis schließen, daß die Plasteinbildung während eines synthetischen Prozesses stattgefunden hat, der am ersten Tage eben der vom Trypsin gleichzeitig bewirkten Proteolyse entsprach; an den folgenden 2 Tagen war die Synthese noch merkbar; nun hatte aber die Proteolyse das Übergewicht.

Was das Ammoniak betrifft, zeigten alle Versuche eine geringe Steigerung, die jedoch nur $0,2-0,3 \%$ des Total-N betrug.

Ferner soll bemerkt werden, daß bei allen Versuchen eine sichtbare Tyrosinausscheidung stattfand.

Versuche mit trypsinverdauten Proteinen.

Bei unseren Versuchen über die Plasteinbildung mit tryptischen Spaltungsprodukten bei Anwendung von Pepsin-HCl und Trypsin benutzten wir folgende Stoffe.

\begin{tabular}{cc|c|c|c}
\hline Stoffe & $\begin{array}{c}\text { Formoltitrier- } \\
\text { barer N }\end{array}$ & Ammoniak-N & $1-(4 \div \mathrm{k})$ \\
\hline Trypsinverdautes Hühnereiweiß & 68,4 & 4,0 & $1-15,1$ \\
, Casein & 60,0 & 7,0 & $1-6,1$ \\
, Witte-Pepton & 52,6 & 4,3 & $1-5,5$ \\
, & Gelatine & 33,6 & 2,4 & $1-6,1$ \\
, Casein & 36,8 & 2,6 & $1-6,7$
\end{tabular}


Die Zubereitung der Versuchsmischungen geschah im wesentlichen wie bei der Anwendung der peptischen Spaltungsprodukte, da sich aber bei der starken Konzentration der Lösungen eine bedeutende Menge von Krystallen ausschied, wurden diese erst durch Kolierung und schwaches Pressen durch Jagonett entfernt, worauf sie in einem Mörser fein gerieben und sodann mit der abkolierten Lösung zu einem feinen Schlamm verrieben wurden, zu dem bezw. Pepsin- $\mathrm{HCl}$ oder Trypsin gesetzt wurde.

Beim Versuch mit Casein vom Spaltungsgrad 36,8 entstand sowohl bei Trypsin wie bei Pepsin eine unbedeutende Plasteinbildung; bei den übrigen Versuchen entstand keine merkbare Plasteinbildung.

Bei den Versuchen mit Pepsin hielt der Formoltiter sich konstant; bei den Versuchen mit Trypsin fand eine Steigerung statt, die bezw. $0,5,0,5,0,4,0$ und $1,2 \mathrm{ccm} 1 / 5-\mathrm{n}-\mathrm{NaOH}$ pro $100 \mathrm{mg} \mathrm{N}$ betrug.

An Ammoniak fand in allen Versuchen eine Steigerung statt.

Diese mit trypsingespaltenen Proteinen angestellten Versuche gaben somit alle ein negatives Resultat. Fernere Versuche über eine etwaige Plasteinbildung tryptischer Spaltungsprodukte können doch möglicherweise ein positives Resultat ergeben. Die Schwierigkeit der Versuche mit tryptischen Spaltungsprodukten liegt darin, daß die schwach trypsingespaltenen Proteine einen Teil koagulablen Proteins und die stark gespaltenen bedeutende Mengen krystallinischer Produkte enthalten, die sich bei der Konzentrierung ausscheiden.

Versuche mit Säurespaltungsprodukten.

Es wurden mit Schwefelsäurespaltungsprodukten folgender Proteine Versuche angestellt: Hühnereiweiß, Casein, Rindfleisch und Witte-Pepton.

Die Spaltungsprodukte wurden durch kurzes Kochen der Proteine mit 25\% igem $\mathrm{H}_{2} \mathrm{SO}_{4}$ (1 Teil Protein und 10 Teile $25 \%$ igen $\mathrm{H}_{2} \mathrm{SO}_{4}$ ) und darauf folgende Entfernung der Schwefelsäure mit Baryumhydroxyd hergestellt. Bei Anwendung dieser Spaltungsprodukte entstand eine typische Plasteinbildung ohne 
Tabelle IX.

Versuche mit Säurespaltungsprodukten in ca. 30\%iger Lösung.

\begin{tabular}{|c|c|c|c|c|c|c|c|}
\hline $\begin{array}{c}\text { An- } \\
\text { gewandte } \\
\text { Stoffe }\end{array}$ & \multicolumn{2}{|r|}{ Versuchsdauer } & \multicolumn{2}{|c|}{$\begin{array}{l}\text { Versuche } \\
\text { mit } \\
\text { Pepsin-HCl } \\
\left.\mathbf{a}^{1}\right) \mid \mathrm{b}\end{array}$} & \multicolumn{2}{|c|}{$\begin{array}{l}\text { Versuche } \\
\text { mit } \\
\text { Trypsin }\end{array}$} & Bemerkungen \\
\hline $\begin{array}{l}\text { Säure- } \\
\text { spaltungs- } \\
\text { produkt } \\
\text { von } \\
\text { Hühner- } \\
\text { eiweiß }\end{array}$ & $\begin{array}{l}3 \\
1 \mathrm{tä} \\
2 \\
4 \\
9\end{array}$ & $\begin{array}{c}\text { Sogleich } \\
\text { Stunden bei } 37^{\circ} \\
\text { igiges Stehen bei } 37^{\circ} \\
>\quad>\quad>37^{\circ} \\
, \quad>37^{\circ} \\
, \quad, 37^{\circ}\end{array}$ & $\begin{array}{c}18,4 \\
- \\
18,5 \\
18,5 \\
18,6 \\
18,3\end{array}$ & $\begin{array}{c}0 \\
3,9 \\
- \\
8,5 \\
10,7 \\
11,4\end{array}$ & $\begin{array}{l}17,2 \\
17,0 \\
17,4 \\
17,3 \\
17,6 \\
18,0\end{array}$ & $\begin{array}{l}- \\
3,4 \\
- \\
- \\
9,0 \\
-\end{array}$ & $\begin{array}{c}\text { Typische } \\
\text { Plasteinbildung } \\
\text { ohne } \\
\text { Gelatinierung. } \\
\text { Bei den Trypsin- } \\
\text { versuchen wurde } \\
\text { etwas Tyrosin } \\
\text { ausgeschieden. }\end{array}$ \\
\hline $\begin{array}{c}\text { Säure- } \\
\text { spaltungs- } \\
\text { produkt } \\
\text { von } \\
\text { Witte- } \\
\text { Pepton }\end{array}$ & $\begin{array}{r}3 \\
1 \text { tä } \\
2 \\
4 \\
9\end{array}$ & $\begin{array}{c}\text { Sogleich } \\
\text { Stunden bei } 37^{\circ} \\
\text { igiges Stehen bei } 37^{\circ} \\
>\quad>\quad>37^{\circ} \\
>\quad>\quad>37^{\circ} \\
>\quad>\quad>37^{\circ}\end{array}$ & $\begin{array}{c}14,4 \\
14,3 \\
14,0 \\
\text { Der K } \\
\text { im Th } \\
\text { stat } \\
\text { gespru }\end{array}$ & $\begin{array}{l}- \\
7,5 \\
12,9 \\
\text { olben } \\
\text { ermo- } \\
\text { en } \\
\text { ingen }\end{array}$ & $\begin{array}{l}13,0 \\
13,0 \\
13,6 \\
13,7 \\
14,3 \\
14,6\end{array}$ & $\begin{array}{l}- \\
6,1 \\
- \\
6,2 \\
6,7 \\
6,8\end{array}$ & desgl. \\
\hline $\begin{array}{c}\text { Säure- } \\
\text { spaltungs- } \\
\text { produkt } \\
\text { von } \\
\text { Rind- } \\
\text { fleisch }\end{array}$ & $\begin{array}{r}3 \\
1 \mathrm{tä} \\
2 \\
4 \\
9\end{array}$ & $\begin{array}{c}\text { Sogleich } \\
\text { Stunden bei } 37^{\circ} \\
\text { agiges Stehen bei } 37^{\circ} \\
, \quad, \quad 37^{\circ} \\
, \quad>37^{\circ} \\
, \quad, 37^{\circ}\end{array}$ & $\begin{array}{l}15,5 \\
15,5 \\
15,5 \\
\text { Der K } \\
\text { im Th } \\
\text { stat } \\
\text { gespru }\end{array}$ & $\begin{array}{l}- \\
5,0 \\
10,3 \\
\text { olben } \\
\text { ermo- } \\
\text { en } \\
\text { ingen }\end{array}$ & $\begin{array}{l}14,2 \\
14,4 \\
15,0 \\
15,3 \\
15,9 \\
16,4\end{array}$ & $\begin{array}{l}- \\
- \\
4,0 \\
6,3 \\
6,2 \\
9,6\end{array}$ & desgl. \\
\hline $\begin{array}{l}\text { Săure- } \\
\text { spaltungs- } \\
\text { produkt } \\
\text { von } \\
\text { Casein }\end{array}$ & $\begin{array}{r}3 \\
1 \text { tă } \\
2 \\
4 \\
9\end{array}$ & $\begin{array}{c}\text { Sogleich } \\
3 \text { Stunden bei } 37^{\circ} \\
\text { dgiges Stehen bei } 37^{\circ} \\
>\quad>37^{\circ} \\
>\quad>37^{\circ} \\
>\quad>37^{\circ}\end{array}$ & $\begin{array}{l}15,4 \\
15,3 \\
15,1 \\
15,1 \\
15,1 \\
15,0\end{array}$ & $\begin{array}{l}- \\
5,1 \\
- \\
10,6 \\
11,9 \\
13,9\end{array}$ & $\begin{array}{l}14,0 \\
14,3 \\
15,1 \\
15,3 \\
15,9 \\
16,6\end{array}$ & $\begin{array}{l}- \\
9,7 \\
8,8 \\
5,2 \\
- \\
5,1\end{array}$ & desgl. \\
\hline $\begin{array}{c}\text { Säure- } \\
\text { spaltungs- } \\
\text { produkt } \\
\text { von } \\
\text { Hühner- } \\
\text { eiweiß }\end{array}$ & $\begin{array}{l}1 \text { tä } \\
3 \\
9\end{array}$ & $\begin{array}{l}\text { Sogleich } \\
\text { ägiges Stehen bei } 370 \\
>\quad>\quad 370^{\circ} \\
>\quad>370^{\circ}\end{array}$ & $\begin{array}{c}18,7 \\
18,7 \\
- \\
18,6\end{array}$ & $\begin{array}{l}- \\
10,9 \\
10,6 \\
14,1\end{array}$ & $\left|\begin{array}{l}16,7 \\
16,8 \\
17,6 \\
18,0\end{array}\right|$ & $\begin{array}{c}- \\
9,4 \\
- \\
10,5\end{array}$ & desgl. \\
\hline
\end{tabular}

1) $\mathrm{a}=$ Formoltitrierung pro $100 \mathrm{mg} \mathrm{N}$ ccm $1 / \mathrm{b}-\mathrm{n}-\mathrm{NaOH} ; \mathrm{b}=$ Plastein- $\mathrm{N}$ in Prozent des Total-N. 
Gelatinierung. Die Formoltitrierung ergab bei Anwendung von Pepsin-HCl einen geringen Ausschlag für eine Synthese, bei Anwendung von Trypsin entstand dagegen eine Spaltung. Die Säurespaltungsprodukte wiesen also ähnliche Verhältnisse auf, wie die peptischen Spaltungsprodukte; die Ausschläge bei Anwendung von Pepsin schwanken aber doch zunächst um den Versuchsfehler, und bei der Anwendung von Trypsin kann man aus den Zahlen nichts darüber folgern, ob die Plasteinbildung hier, außer von einer Proteolyse, zugleich von einer Synthese begleitet wird, wie dies beim Versuch mit pepsingespaltenem Hühnereiweiß und Trypsin (Tabelle VIII) der Fall war.

Tabelle IX enthält eine Übersicht über die Versuchsresultate.

Bei den Versuchen mit Trypsin fand eine geringe Tyrosinausscheidung statt.

Versuche mit Alkalispaltungsprodukten.

Es wurden mit 2 Alkalispaltungsprodukten von Hühnereiweiß Versuche angestellt; die Spaltungsprodukte wurden durch 4wöchiges Stehenlassen einer $2 \%$ igen Lösung von Hühnereiweiß bezw. in $1 / 10-n-$ und $3 / 10-n-N a O H$ im Thermostaten hergestellt.

Die Versuchsmischungen wurden in gewöhnlicher Weise hergestellt, indem die Mischungen mit Salzsäure neutralisiert und vor dem Eindampfen filtriert wurden. Beim Eindampfen schieden sich NaCl-Krystalle aus, die durch Kolierung durch Jagonett entfernt wurden, und beim Zusatz von Salzsäure in den Versuchen mit Pepsin schied sich ein Teil alkaligespaltenen Albumins aus, das gleichfalls vor dem Zusatz von Ferment entfernt wurde.

Die angewandten Spaltungsprodukte enthielten bezw. 23,2 und $34,7 \%$ formoltitrierbaren $\mathrm{N}$, wovon $10,0-10,3 \%$ Ammoniak- $\mathrm{N}$ waren. $1-(4 \div \mathrm{k})$ betrug bezw. $1-3,7$ und 1-4,3.

Bei Anwendung von Pepsin-HCl entstand eine typische Plasteinbildung ohne Gelatinierung. Die Plasteinausfällung betrug im Laufe von 19 Tagen bezw. 6,7 und 8,6\% des Total-N, und 
gleichzeitig fand eine Vermehrung des formoltitrierbaren $\mathrm{N}$ statt, die pro $100 \mathrm{mg} \mathrm{N}$ bezw. 0,8 und $0,3 \mathrm{ccm}^{1 / 5}-\mathrm{n}-\mathrm{NaOH}$ betrug.

Bei Anwendung von Trypsin entstand keine merkbare Plasteinbildung, wohl aber in beiden Fällen eine Vermehrung des formoltitrierbaren $\mathrm{N}$, die $1,0 \mathrm{ccm}{ }^{1 / 5}-\mathrm{n}-\mathrm{NaOH}$ pro $100 \mathrm{mg} \mathrm{N}$ entsprach, und gleichzeitig eine sichtbare Tyrosinausscheidung.

Das Ammoniak hielt sich sowohl bei den Versuchen mit Pepsin als bei denen mit Trypsin konstant.

Resumé.

\begin{tabular}{|c|c|c|}
\hline & Mit Pepsin-HCl & Mit Trypsin \\
\hline $\begin{array}{l}\text { Peptische } \\
\text { Spaltungs- } \\
\text { produkte. }\end{array}$ & $\begin{array}{l}\text { 1. Typische Plasteinbildung } \\
\text { mit oder ohne Gelati- } \\
\text { nierung. } \\
\text { 2. Deutlicher Ausschlag für } \\
\text { Synthese. } \\
\text { 3. Der Prozeß geht bei Ver- } \\
\text { dünnung wieder zurück. }\end{array}$ & $\begin{array}{l}\text { 1. Typische Plasteinbildung } \\
\text { ohne Gelatinierung. } \\
\text { 2. Von einer Proteolyse und } \\
\text { wahrscheinlich gleich- } \\
\text { zeitig von einer Synthese } \\
\text { begleitet. } \\
\text { 3. Tyrosinausscheidung. }\end{array}$ \\
\hline $\begin{array}{c}\text { Tryptische } \\
\text { Spaltungsprodukte } \\
\text { (stark gespalten). }\end{array}$ & $\begin{array}{l}\text { 1. Keine Plasteinbildung. } \\
\text { 2. Proteolytische Spaltung. }\end{array}$ & $\begin{array}{l}\text { 1. Keine Plasteinbildung. } \\
\text { 2. Proteolytische Spaltung. }\end{array}$ \\
\hline $\begin{array}{c}\text { Säure- } \\
\text { spaltungsprodukte }\end{array}$ & $\begin{array}{l}\text { 1. Typische Plasteinbildung } \\
\text { ohne Gelatinierung. } \\
\text { 2. WahrscheinlichSynthese. }\end{array}$ & $\begin{array}{l}\text { 1. Typische Plasteinbildung } \\
\text { ohne Gelatinierung. } \\
\text { 2. Proteolytische Spaltung. } \\
\text { 3. Tyrosinausscheidung. }\end{array}$ \\
\hline $\begin{array}{c}\text { Alkali- } \\
\text { spaltungsprodukte. }\end{array}$ & $\begin{array}{l}\text { 1. Typische Plasteinbildung } \\
\text { ohne Gelatinierung. } \\
\text { 2. Proteolytische Spaltung. }\end{array}$ & $\begin{array}{l}\text { 1. Keine Plasteinbildung. } \\
\text { 2. Proteolytische Spaltung. } \\
\text { 3. Tyrosinausscheidung. }\end{array}$ \\
\hline
\end{tabular}

Das Ammoniak nimmt bei keinem der Versuche ab und kann somit nicht an der Synthese teilnehmen. 